\title{
Histopathological IMAgE ANALYSIS USING IMAge Processing TeCHNIQUES: AN OVERVIEW
}

\author{
A. D. Belsare ${ }^{1}$ and M. M. Mushrif ${ }^{2}$ \\ ${ }^{1,2}$ Department of Electronics \& Telecommunication Engineering, Yeshwantrao Chavan \\ College of Engineering, Hingna Road, Nagpur, MH, India \\ amolibelerediffmail.com \\ milindmushrif@yahoo.com
}

\begin{abstract}
This paper reviews computer assisted histopathology image analysis for cancer detection and classification. Histopathology refers to the examination of invasive or less invasive biopsy sample by a pathologist under microscope for locating, analyzing and classifying most of the diseases like cancer. The analysis of histoapthological image is done manually by the pathologist to detect disease which leads to subjective diagnosis of sample and varies with level of expertise of examiner. The pathologist examine the tissue structure, distribution of cells in tissue, regularities of cell shapes and determine benign and malignancy in image. This is very time consuming and more prone to intra and inter observer variability. To overcome this difficulty a computer assisted image analysis is needed for quantitative diagnosis of tissue. In this paper we reviews and summarize the applications of digital image processing techniques for histology image analysis mainly to cover segmentation and disease classification methods.
\end{abstract}

\section{KEYWORDS}

Image processing, histopathological image analysis, image segmentation, and computer assisted diagnosis.

\section{INTRODUCTION}

Histology deals with the study of the microscopic structure of cells and tissues of organisms. The knowledge of biological (microscopic) structures and their functions at the sub-cellular, cellular, tissue and organ levels is central to the study of disease proliferation and prognosis of disease. Also to study and analyze histological image under microscope, pathologists identify the morphological characteristics of tissue which indicates the presence of disease like cancer. The biopsy sample is processed and its sections are placed onto glass slides to observe them under microscope for analysis. Pathologist examine the tissue slides under a microscope and observe it at various magnification levels such as $10 \mathrm{X}, 20 \mathrm{X}, 40 \mathrm{X}, 100 \mathrm{X}$ etc. to view cells, glands, nucleus, and detects the resemblance of these structures with normal vs. diseased tissue. If the disease detects the grading process is performed which deals with spreading of infected cells all over the tissue. Then for each patient the prognosis and further treatment is planned by considering grade of disease.

This diagnosis by pathologist is subjective and prone to inter, intra observer variations. Therefore a quantitative assessment of these images is very essential for objective diagnosis. Also, now a day's because of development in digital scanners for pathological image acquisition provide us the digital image data for computer assisted image analysis using digital image processing algorithms. Thus, computer assisted disease diagnosis (CAD) plays a very important role and has become a major research subject in histopathological imaging and diagnostic where

DOI : $10.5121 /$ sipij.2012.3403 
Signal \& Image Processing : An International Journal (SIPIJ) Vol.3, No.4, August 2012

different image processing techniques can be used to analyze these images for disease diagnosis and prognosis. Therefore, histology provides a scientific foundation for clinical research, education, and practice.

The aim of this paper is to investigate robust and accurate image analysis algorithms for computer-assisted interpretation of histopathology imagery. Different image processing techniques will be applied for image texture classification, gland \& nuclei segmentation, cell counting, cell type identification or classification to deriving quantitative measurements of disease features from histological images and automatically determine whether a disease is present within analyzed samples or not. Also this research will help to decide the different grades or severity of disease if the disease is present in the sample.

Computer aided histopathological study has been conducted for various cancer detection and grading applications, including prostate (Naik et al., 2007, Doyle et al., 2007), breast (Doyle et al., 2008, Naik et al., 2008, Jean-Romain Dalle et al., 2008), renal cell carcinoma (S. Waheed et al., 2007) pediatric tumor neuroblastoma (Olcay S et al., 2009) and lung (Kayser et al., 2002) cancer grading. Using different segmentation, feature extraction and classification techniques the researchers analyzed histopathology images. Here in this paper the different image processing techniques on histopathological images specially breast cancer histopathology analyses are reviewed.

The paper is organized to discuss the need for, and analyze the procedure for computer aided histopathology image segmentation and classification. These analysis procedures are also applicable to all image modalities in medical image analysis like ultrasound, MRI, CT Scan etc. In section 2, basic histology image analysis flow and the procedure for preparation of histopathology slides for microscopic analysis including the image magnification/resolution needed for various analyses is described. Further in section 3, segmentation techniques for histopathological images are reviewed. Section 4, includes brief review about the feature extraction and selection for different segmented results. Finally, the overview about classification methods for histopathology image with the future analysis in the related field is discussed in section 5. Followed by, the comparison and conclusion on the study is given. In literature one can find large number of applications in microscopic image analysis feature extraction and selection along with many image processing methods for preprocessing, segmentation, and classification, here only some examples are presented.

\section{Basic Histopathological Image Analysis: Overview}

After acquiring digital histology image through biopsy sample, the manual examination of images leads to variability in diagnosis. To overcome this problem, computer assisted systems are employed which gives objective analysis of diseases. The basic steps required for implementing computer assisted analysis system are as shown in Figure 1 below. This consists of digital image processing techniques such as image segmentation, feature extraction, classification etc. 

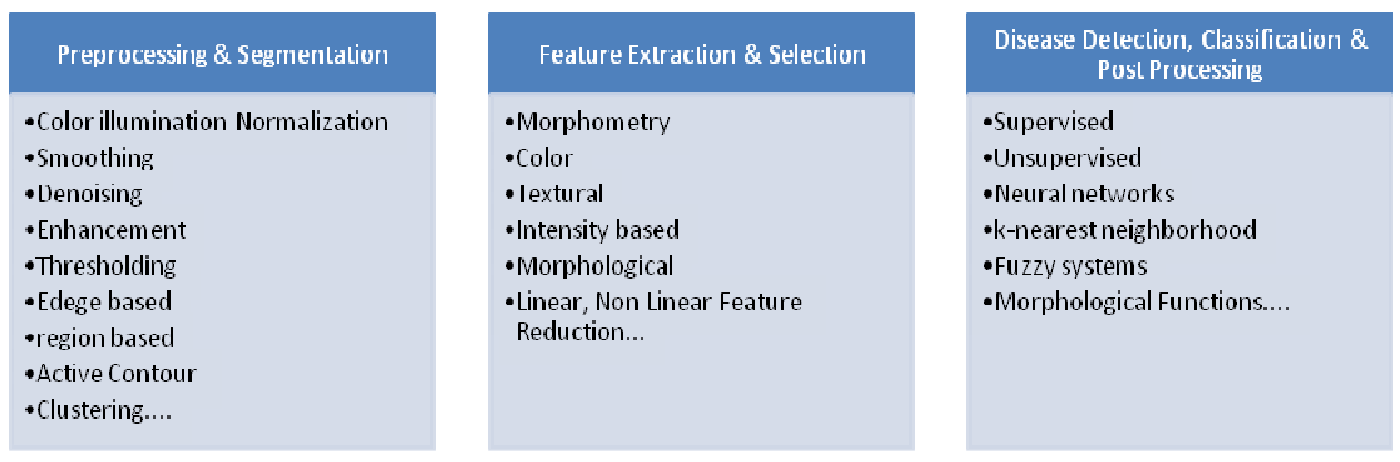

Figure 1: Overview of histopathological image analysis

\subsection{Histology image analysis}

The histopathological image analysis includes the computations performed at different magnifications $(\times 2, \times 4.5, \times 10, \times 20$, and $\times 40)$ for multivariate statistical analysis, diagnosis and classification. It can be done at lower magnification for tissue level analysis. As Cigdem Demir et al. [1] discussed on both tissue level and cell level analysis methods for cancer diagnosis. They analyzed histopathology images using image preprocessing, feature extraction and classification techniques such as thersholding, morphological processing, region based, and boundary based, and supervised classification techniques. The recent advancement of 'digital pathology' needs development of quantitative and automated computerized image analysis algorithms to assists pathologist in interpreting the large number of digitized histopathological images. Computer aided diagnosis algorithms have been proposed for detecting neuroblastoma [11], also computer aided renal cancer image analysis based on Bayesian classifier, $\mathrm{k}$ means algorithm is proposed by S. Waheed et al. [2] to improve inter, intra- observer variability in decision by pathologist. Total $98 \%$ classification accuracy with cross validation was achieved by using high magnification images at $200 \mathrm{X}$ magnification for system development. O. Sertel et al. [3] proposed an image analysis method on neuroblastoma histopathological images based on likelihood function estimation. The cancer cells were detected with sensitivity of $81.1 \%$ using component-wise-2 step thresholding. Similarly, in the literature one can find applications of such different image analysis methods for classification of histopathology image data. These methods can also be applied to the breast cancer histopathology images for their quantitative analysis and evaluation [4].

Depending on the application or kind of disease the image processing steps may vary, but in general the image processing algorithms are similar for most of the applications. Jun Xu et. al. [5] proposed breast cancer histopathology color image boundary based segmentation using geodesic active contours (GAC) and weighted mean shift normalized cut. It involves defining a color swatch from image and apply mean shift normalized cut algorithm as initial segmentation for detection of initial object boundary. Then using GAC edge detection based color gradient final segmentation results were obtained. Total 60 digitized histopathology slides were used and (WNCut-CGAC) results were compared with Chan Vese model. The percentage accuracy in sensitivity, specificity was found to be improved with WNCut-CGAC model with robust initialization having minimum user interaction.

The different algorithms developed for histology analysis are summarized in Table 1 given below. 
Signal \& Image Processing : An International Journal (SIPIJ) Vol.3, No.4, August 2012

Table 1: Different algorithms for histopathological image analysis

\begin{tabular}{|c|c|c|}
\hline $\begin{array}{l}\text { Authors, Year of } \\
\text { Publications }\end{array}$ & Organ & $\begin{array}{l}\text { Method for Segmentation, Classification of } \\
\text { Histopathology Images }\end{array}$ \\
\hline $\begin{array}{l}\text { Ajay Basavanhallya } \\
\text { et. al. } 2011\end{array}$ & Breast & $\begin{array}{l}\text { Hierarchical Normalized cut, Color Gradient } \\
\text { Active Contour [11] }\end{array}$ \\
\hline Jun Xu et. al. 2011 & Prostate & Geodesic Active Contour [5] \\
\hline S. Olcay et. al. 2008 & $\begin{array}{l}\text { Follicular } \\
\text { Lymphoma }\end{array}$ & $\begin{array}{l}\text { Texture Classification using Non Linear color } \\
\text { Quantization, Self Organizing Maps [18] }\end{array}$ \\
\hline Omar Khadi, 2010 & $\begin{array}{l}\text { Meningioma } \\
\text { Tumor }\end{array}$ & Texture Classification using fractal features [16] \\
\hline $\begin{array}{l}\text { C. Demir et. al. , } \\
2010\end{array}$ & $\begin{array}{l}\text { Colon } \\
\text { Glands }\end{array}$ & Segmentation using Object graph approach [13] \\
\hline $\begin{array}{l}\text { Muthu Rama et. al., } \\
2009\end{array}$ & Oral Mucosa & SVM, Classifier [22] \\
\hline $\begin{array}{l}\text { Hui Kong et. al, } \\
2011\end{array}$ & $\begin{array}{l}\text { Follicular } \\
\text { Lymphoma }\end{array}$ & Color Texture cell Segmentation [28] \\
\hline $\begin{array}{l}\text { M. Dundar et. al., } \\
2011\end{array}$ & Breast & Gaussian Mixture Model based segmentation [26] \\
\hline $\begin{array}{l}\text { Akif Tosum et. al. } \\
2011\end{array}$ & Breast & $\begin{array}{l}\text { Graph Run length matrices for image segmentation } \\
\text { [27] }\end{array}$ \\
\hline
\end{tabular}

\subsection{Preparation of histological tissue slide}

Histology is nothing but the microscopic examination of a biopsy specimen which is processed and fixed onto glass slides. To examine different architecture and components of a tissue under microscope, the gross sections are made with wax, and dyed with one or more stains. Staining is used by pathologists to separate cellular components for structural as well as architectural analysis of tissue for diagnosis. Most commonly Hematoxylin -Eosin (H \& E) stain is used which separates cell nuclei, cytoplasm and connective tissue. Hematoxylin stains cell nuclei blue, whereas Eosin stains cytoplasm and connective tissue pink. The other stains are DAB, immune-histochemistry stain etc. 
The image were acquired using Motic B1 series system microscope at 10X magnification with $10 \mu \mathrm{m} /$ pixel calibration and $40 \mathrm{X}$ with $2.5 \mu \mathrm{m} /$ pixel calibration. Here the analysis of $\mathrm{H} \& \mathrm{E}$ stained images only will be considered for avoiding different staining effects on final result. The images are as shown in Figure 3 and Figure 4 which show low and high magnified normal and malignant breast cancer images.

The steps in preparation of tissue slide are as sown in Figure 2 and it includes:

a. Fixing: Samples of biological tissue/ gross are "fixed" with chemical fixation to preserve the cells/tissue.

b. Processing: Tissue processing is essential to remove water from the gross tissue (dehydration) and replace it with a medium which solidifies it. This helps to cut thin sections of sample

c. Embedding sample in wax: The result of embedding hardened wax blocks contains the original biological samples together with other substances in complete preparation process.

d. Sectioning: Sectioning an embedded tissue sample is the step necessary to produce sufficiently thin slices of sample that the detail of the microstructure of the cells/tissue can be clearly observed using microscopy techniques. Then transfer the thin cut of sample on to a clean glass slide.

e. Staining: Finally, the mounted sections are treated with an appropriate histology stain. Staining biological tissues is done to both increase the contrast of the tissue and also highlight some specific features of interest - depending on the type of tissue and the stain used.

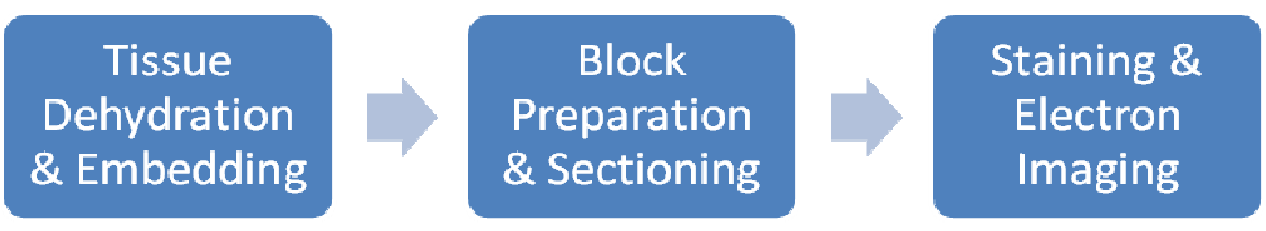

Figure 2. Basic Steps for Preparation of Histopathology Samples.

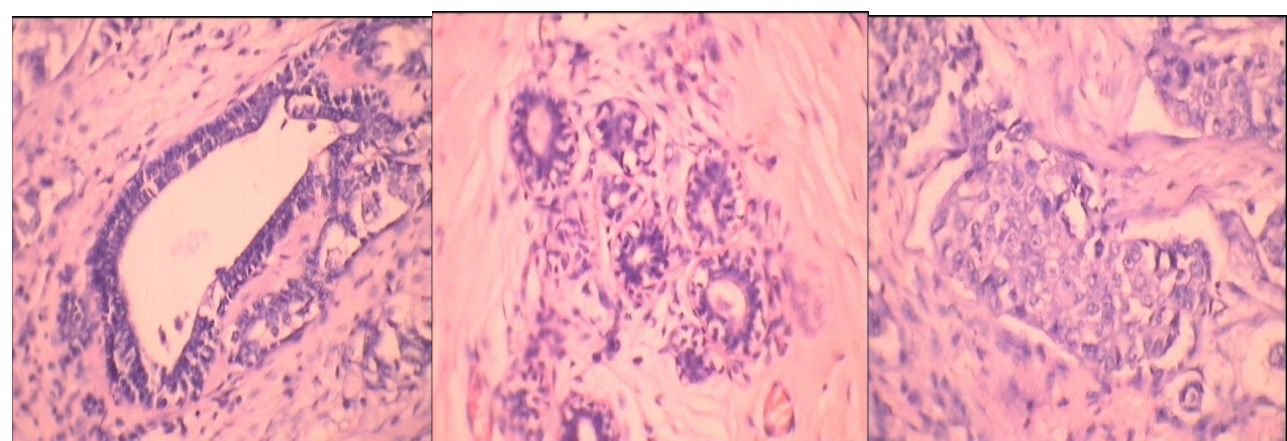

a.

b.

c.

Figure 3. Histopathological H \& E Stained Breast Cancer Images received from GMCH, Nagpur a. and b. normal duct and lobules, c. malignant breast cancer image at 40X 
Signal \& Image Processing : An International Journal (SIPIJ) Vol.3, No.4, August 2012

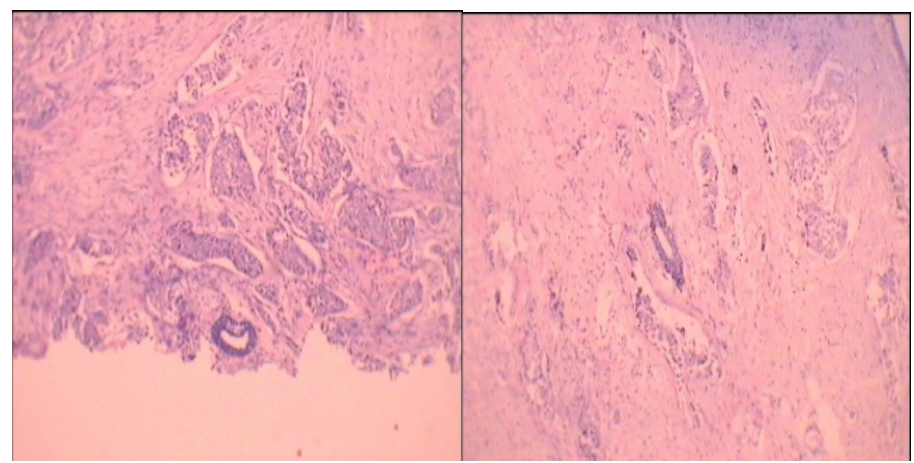

a.

b.

Figure 4. Histopathological H \& E Stained Breast Cancer Images received from GMCH, Nagpur a. and b. normal and malignant both at $10 \mathrm{X}$

\section{Histopathology Image Segmentation}

The diagnosis of disease or its grading in histopathology images deals with identification of histological structures like cancer cell nuclei, glands, lobule formation as in case of breast cancer. The other morphological appearance of these structures like size, shape, and color intensity, are also important factors for presence of disease. To analyze all these indicators histopathology images firstly should be segmented. For such segmentation approaches the images should be acquired at various magnification levels such as for nucleus segmentation $40 \mathrm{X}$, cell identification with 20X, where as gland, tissue segmentation requires $10 \mathrm{X} / 4 \mathrm{X}$ magnified histology image. We can consider the image analysis for low power analysis (10X) or high power analysis (40X). After aquicision, histopathological images are true color images, so to interpret these images on computer one has to observe artifacts in the image because of staining procedure. To eliminate the effect of such noise in the image it should be preprocessed, de-noised and enhance the image quality to get good segmentation and classification results. This includes color normalization if color image is processed for cell morphology analysis, image de-noising, enhancement to get more detailed cell or structure information from the image etc. Also it is essential to remove the effect of variation in histology image as the histology images are stained with different staining proportions and scanning conditions hence there may be color variations in image which affects the results.

After preprocessing, image segmentation is one of the most important steps of the automatic medical diagnosis based on the analysis of the microscopic images, and is also a difficult task to diagnose the disease correctly. Image segmentation separates objects of interest from background through various methods in image processing such as thresholding where the intensity value is used to separate the regions. In histology this can mainly be used for detection of nuclei, stroma and background [33, 35].Due to complex nature of pathological images, standard segmentation methods or modified versions of them like thresholding, Hidden Markov model, watershed algorithm, active contours, cellular automata, Grow- Cut technique, as well as new approaches like fuzzy sets of I and II type, seeded region growing can be used for cell identification and classification $[11,12,13,29,30,34]$. Through the segmentation one can choose the area of interest (AOI) in an image like cell, nuclei, tumor etc. in case of pathology images for further analysis. The segmentation on basis of region is performed if tumor is to be consider, boundary based or methods based on thresholding are used for identification of cell structure from background area as an object in an image, as Yousef Al-Kofahi, et.al. [14], proposed a method for segmenting cell nuclei with image foreground using a graph-cuts-based binarization and multiscale Laplacian-of-Gaussian filtering constrained by distance-map-based adaptive scale selection for the nuclear seed points detection. Also in the cytological image 
analysis, segmentation is the first step; the Hough transform with the watershed algorithm is used for this by the researchers for automatic nuclei localization [15].

The texture approach for finding presence of disease in image sample for different cases in microscopic imagery is also very helpful, Omar S. Al-Kadi, [16] proposed a texture based technique to classify meningioma tumors correctly based on optimum texture measure combination, which inspects the separability of the RGB color channels and selects the channel which best segments the cell nuclei of the histopathological images and a Bayesian classifier was used for meningioma subtype discrimination. Overall classification accuracy of $92.50 \%$ was achieved. From the literature we can found that pattern classification techniques such as support vector machines (SVM) and radial basis function neural network (RBFNN) can also help to improve the diagnosis in cytological image analysis. These networks are used to extract the patterns and one can compare the use of polynomial kernels in ascertaining the diagnostic accuracy of cytological data obtained from the breast cancer tumors i.e. benign and malignant. With different performance measures they evaluated and compared the qualities of these classifiers [17]. Texture features can also be used for tissue segmentation based on gray level run length matrices (GRLM). The Akif Burak Tosum et.al.[27] introduced the GRLM texture based features for color images and found high segmentation accuracies with it. The color histopathological images of colon tissues at 5X magnification were used in the study and then texture features were extracted from color graph run length matrix. Also the color texture features can be calculated using local neighborhood of each pixel in image with local Fourier Transform applied on new color space. An efficient LFT extraction algorithm based on image shifting and image integral is used by Hui Kong et.al.[28] and using above approach achieves good segmentation results for lymphoma grading with 5.25\% total error rate. A variety of computational intelligence approaches to nuclei segmentation in the microscope images is presented the literature $[6,18]$.

There is also a Region based approach for histopathological image segmentation namely, seeded volume growing, constrained erosion-dilation techniques and 3-D watershed algorithm on 3-D histo-pathological images. These methods are evaluated and the results are compared with some modification in [19]. M. MuthuRama Krishnan, et.al. presents computer aided system for segmentation and classification of sub-epithelial connective tissue (SECT) cells in oral mucosa of normal and oral sub-mucous fibrosis (OSF) conditions [22]. Segmentation has been carried out using multi-level thresholding and subsequently the cell population has been classified using support vector machine (SVM) based classifier. The analysis and diagnosis of microscopic images is carried out for the disease like cancer where the interpretation of samples is very important to detect the presence of disease and further treatment for patient. Some of the researchers worked on such application related with breast, prostate, lung cancer [20, 21].

Baouchan Pang et al [8], proposed machine learning technique for cell nucleus segmentation of color histological image based on convolutional networks. Using gradient descent technique, convolutional network with 3 hidden layers and 8 feature maps per hidden layer was trained for segmenting cell from background. The gradient descent technique is utilized to optimize the network. They used L*a*b* and RGB color space for segmentation algorithms. They found that this method gives more promising results where inter-image variability in staining is involved. [23] To segment individual cell, firstly the cytological components in tissue should be separated such as, cytoplasm, extra cellular material, RBC, and background. In H\&E stained tissue samples, nuclear and cytoplasm regions have blue color where as RBCs are red and stroma region in pink color. A computer assisted system using mean shift algorithm is used to segment cytoplasm and cell nuclei in histopathological image. 89\% accuracy is achieved to detect malignant cancer cell through this approach.

Identification of tubules and cancer nuclei is one of the important criterions in detection of breast cancer. Ajoy Basavanhally et. al. [4], detects tubule and cancer nuclei using O'Callaghan neighborhood and Color Gradient based Active Contour model (CGAC) through color 
deconvolution scheme as shown in Figure 5. Here an automated detection of tubules using spatial proximity with low level structures like lumen, cytoplasm and nuclei is presented. By using graph based image features lumen area was detected and segmented with $86 \%$ accuracy. It was compared with manual grading of tubular density and $89 \%$ accuracy was achieved.

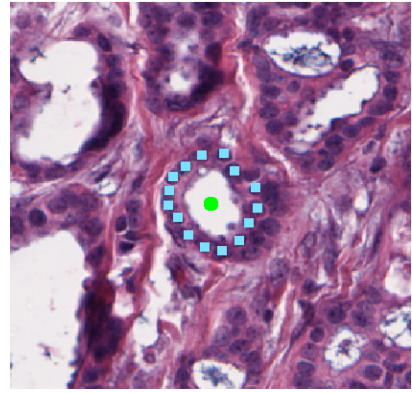

a.

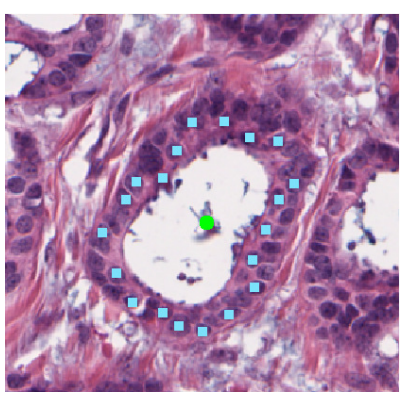

b.

Figure 5. True lumen area in a. Small tubule \& b. large Tubule present in breast cancer tissue figures from [4].

Also the shape based segmentation methods can also be used for gland or nuclei segmentation. Like an expectation-maximization (EM) algorithm was used for automatically detecting the centers of lymphocytes in breast cancer histology images. Initially contours were detected using partial differential equation with centers [6]. In Naik et. al. [9] a level set method for gland segmentation for prostate and a breast cancer histology image is presented. A level set was initialized by the likelihood scenes generated by Bayssian classfier.

Also histology images could be segmented based on color texture and homogeneity. Color and texture information in image could be combined with consideration of the distribution of components in tissue image the histology images can be segmented. In [32] the researcher used new object-oriented textural segmentation for detecting colon cancer. With the new approach for homogeneity measure they achieved $94.89 \%$ accuracy to segment normal and cancerous regions in colon histology images.

\section{Feature Extraction}

After segmentation, features are extracted either at the cellular or at the tissue-level to measure morphological characteristics of image for abnormality or to classify the image for different grades of disease. The cellular-level features focuses on quantifying the properties of individual cells without considering spatial dependency between them. For a single cell, the morphological, textural, fractal, and/or intensity-based features can be extracted. The tissuelevel features quantify the distribution of the cells across the tissue; for that, it primarily makes use of either the spatial dependency of the cells or the gray-level dependency of the pixels. For a tissue, the textural, fractal, and/or topological features can be extracted as shown in Table 2. The aim of the diagnosis step is (i) to distinguish benignity and malignancy or (ii) to classify different malignancy levels by making use of extracted features. This step uses statistical analysis of the features and machine learning algorithms to reach a decision.

Scot Doyel et al. [10] used textural and nuclear architectural features for analysis of breast cancer histopathology image. Spectral clustering is used to reduce the dimensionality of the feature set. The different types of feature extraction techniques are textural features using grey level, Haralick features, graphical features, and Gabour filter features, Graph features using Voroni diagram, Delauny Triagulation, Minimum Spanning Tree, Nuclear Features, morphological features, Topological features etc. $[1,9,10,16,24,25]$. These can be used for 
Signal \& Image Processing : An International Journal (SIPIJ) Vol.3, No.4, August 2012

analysis of histology image as 1) normal or abnormal image, 2) for identifying grades of cancer, 3) also these features can be used to segment the tissue based on architectural information obtained from graph based features.

Table 2: Different Features for Analysis

\begin{tabular}{|l|l|}
\hline $\begin{array}{l}\text { Textural Features: First order statistics, } \\
\text { GLCM, Run length matrix }\end{array}$ & $\begin{array}{l}\text { smoothness, coarseness, regularity, correlation, } \\
\text { contrast and angular second } \\
\text { moment for the tissue classification }\end{array}$ \\
\hline $\begin{array}{l}\text { Graph Features: Voronoi Diagram, } \\
\text { Delauny triangulation, Minimum spanning } \\
\text { tree }\end{array}$ & $\begin{array}{l}\text { Number of nodes, edges, area, roundness } \\
\text { factor, edge length, spectral radius to detect } \\
\text { tubules or cells }\end{array}$ \\
\hline Morphological features of cell & $\begin{array}{l}\text { Radius, area, perimeter, size, shape, roundness, } \\
\text { smoothness to segment cell boundary for } \\
\text { abnormality detection }\end{array}$ \\
\hline
\end{tabular}

\section{Classification}

For histopathology imagery, the choice of classifier is also very important to deal with large, highly dense dataset. After segmentation and feature extraction, selection the different classifiers are used to classify images for diagnosing abnormality in image. In this step, a cell or tissue is assigned to one of the classes and then it can also be classified for malignancy level i.e. grading of disease. A lot of work is done on the classification of histopathological images using machine learning algorithms like neural network, k-nearest neighborhood algorithm, logistic regression method, fuzzy systems etc. Muthu Rama et. al. [22] used SVM approach to classify oral mucosa histology images. A Bayesian classifier is implemented based on the defined feature space for characterizing inflammatory and fibroblast cells in order to observe the cell distribution in healthy state. The performance of this system is evaluated with $97.19 \%$ overall classification accuracy [24]. Along with Bayesian Classifier the AdaBoost Algorithm is also used for Gleason grading with textural feature extraction [25]. Intraductal Breast Lesions are classified using expectation maximization and watershed transform by M.Murat Dundar et.al.[26]. In this work the system for automatic diagnosis of preinvasive breast images is developed with 62 patient cases and the overall classification accuracy for classification is $87.9 \%$ and the result was tested with the diagnostic accuracy of pathologist on same data set.

A support vector machine (SVM) classifier is used (1) to distinguish between cancerous and non-cancerous images, and (2) to distinguish between images containing low and high grades of cancer $[10,22]$. The system achieves a 95.8\% accuracy in distinguishing cancer from noncancer using texture-based characteristics (Gabor filter features), and 93.3\% accuracy in distinguishing high from low grades of cancer using architectural features.

In recent years the researchers are using, object location for tissue representation instead of spatial representation of tissue and structural features from color graphs are used for automated colon cancer diagnosis which gives more promising results (82.65\% test accuracy) as they are considering the location and structure of tissue in histology images [31]. Here the homogeneous colon cancer images are used to obtain better results over intensity based textural approaches.

\section{Comparison}

Compared with radiological image processing and analysis, histology image processing and analysis is emerging area having more challenging problems. Histology images have very complicated structure than radiology images. The study on different aspects of histopathological image analysis leads to very promising results. But the comparison of different methodologies 
Signal \& Image Processing : An International Journal (SIPIJ) Vol.3, No.4, August 2012

applied to histology images is difficult since each research uses different image dataset and gives different metrics to show results. Each study developed the automated system by using different segmentation, features and classification algorithms. Even the images at different magnification are used according to the aim of study. Also the tissue architecture and cell are different for different organs; hence the method applied to one organ may not work on another. Some of studies also differentiated only normal and abnormal images, whereas in some papers the images are classified for different grades of disease. Low and high grade cancer can be detected using different texture features for tissue image. Variation in normal gland structure, size and shape of nuclei are the measure of identifying presence of cancer and its grade. Further histopathology analysis by pathologist is subjective because of inter and intra observer variability. This causes variation in classification results when compared manually. To obtain good computational results one has to use large image data base.

In recent years color graphs and its texture features are used for tissue segmentation and classification which improves the result over conventional pixel distribution method for the histology image analysis. This could be very effective as the histology image is much complicated for segmentation as the color and distribution of pixel changes with staining and thus using spatial relation between objects for object oriented segmentation good results are achieved. SVM is most commonly used classifier for classification of histology images for cancer diagnosis. A comparative analysis for the segmentation and feature extraction for classification algorithms for histology images has been made and tabulated as shown in Table 3 and Table 4 given below:

Table 3: Comparative assessment for histology image analysis methods

\section{Segmentation of gland structure in histology images based on color texture}

Pixel based: Gives good segmentation results for the images with regular gland structure but can give incorrect results with variation in normal gland structure and presence of artifacts due to staining variation, fixation, sectioning of tissue and false positive results due to presence of fat, stroma, and lumen like areas. $[1,3,5,6,11,26$, 27,28 etc.]

Object based: Improves segmentation accuracy compared to pixel based algorithms for histology images by considering organization of gland structure as an object. [4, $13,27,31,32]$

Classification of histology images using texture/architecture features

Texture features with self organizing map: classifies high grade and low grade follicular lymphoma with average of $90.30 \%$ accuracy. $[7,18]$

Fractal features: Calculates size and shape features for cell nuclei and classifies breast and prostate cancer with $87.9 \%$ and $96.7 \%$ classification accuracy.[2,8, 9, 16]

GLCM/GRLM features: Can be used as texture descriptors for classification and detection of normal structure of gland or tissue in spite of variations in appearance of tissue in histology image. [1, 16, 27, 32]

Graph features: Mostly used for object based segmentation and detecting architectural features of histology image along with texture features to improve classification and segmentation accuracy. These features measure the spatial arrangement of all objects in the image. $[1,9,10,16,24,25]$ 
Table 4: Histology image analysis for different methods

\begin{tabular}{|c|c|c|c|}
\hline $\begin{array}{l}\text { Method for Segmentation, } \\
\text { Classification of } \\
\text { Histopathology Images }\end{array}$ & Organ & Results & Problem with method \\
\hline $\begin{array}{l}\text { Hierarchical Normalized } \\
\text { cut , Color Gradient Active } \\
\text { Contour [11] }\end{array}$ & Breast & $\begin{array}{l}\text { Achieves } 89 \% \\
\text { segmentation accuracy }\end{array}$ & $\begin{array}{l}\text { Detects false positive } \\
\text { errors due to presence of } \\
\text { lumen like areas. }\end{array}$ \\
\hline $\begin{array}{l}\text { Texture Classification } \\
\text { using Non Linear color } \\
\text { Quantization, Self } \\
\text { Organizing Maps [18] }\end{array}$ & $\begin{array}{l}\text { Follicular } \\
\text { Lympho } \\
\text { ma }\end{array}$ & $\begin{array}{l}\text { Results in } 85.5 \% \\
\text { average accuracy }\end{array}$ & $\begin{array}{l}\text { Staining variation may } \\
\text { affect the result }\end{array}$ \\
\hline $\begin{array}{l}\text { Texture Classification } \\
\text { using fractal features [16] }\end{array}$ & $\begin{array}{l}\text { Meningio } \\
\text { ma } \\
\text { Tumor }\end{array}$ & $\begin{array}{l}\text { Gives } 92.5 \% \text { accuracy } \\
\text { using individual texture } \\
\text { measure for classifying } \\
\text { meningioma tissue. }\end{array}$ & $\begin{array}{l}\text { Gives misclassification } \\
\text { results due to non } \\
\text { homogeneity cell structure. } \\
\text { Segmentation separability } \\
\text { assessment is required in } \\
\text { order to select the } \\
\text { optimum color channel }\end{array}$ \\
\hline $\begin{array}{l}\text { Segmentation using Object } \\
\text { graph approach [13] }\end{array}$ & $\begin{array}{l}\text { Colon } \\
\text { Glands }\end{array}$ & $\begin{array}{l}87.59 \% \text { accuracy for } \\
\text { homogeneous images } \\
\text { and improves accuracy } \\
\text { over pixel base } \\
\text { algorithm. }\end{array}$ & $\begin{array}{l}\text { Needs parameter } \\
\text { optimization which } \\
\text { decreases segmentation } \\
\text { results }\end{array}$ \\
\hline $\begin{array}{l}\text { Graph Run length matrices } \\
\text { for image segmentation } \\
\text { [27] }\end{array}$ & Breast & $\begin{array}{l}\text { New texture descriptor } \\
\text { for unsupervised } \\
\text { classification. } 99.0 \% \\
\text { accuracy with Graph } \\
\text { RLM texture } \\
\text { segmentation even for } \\
\text { variation in appearance } \\
\text { of gland. }\end{array}$ & $\begin{array}{l}\text { Computational complexity } \\
\text { depends on number of } \\
\text { primitives in image }\end{array}$ \\
\hline
\end{tabular}

\section{Conclusion}

In this review, we studied different steps to automatically analyze histopathological images for objective diagnosis. This automated analysis assists pathologist in diagnosis and lessen their time for reviewing large number of tissue slide per day. The digital histopathological images are acquired through computerized electron microscope after tissue slide preparation. Different magnification images are used for different types of analysis; like for tissue classification low magnification (10X) and for cell segmentation and analysis higher magnification (40X). For computer aided analysis there are mainly three steps: Segmentation, Feature Extraction and Classification. The algorithms developed for automated analysis and evaluation of histology images assists the pathologists in disease diagnosis and also reduces human error. 
Signal \& Image Processing : An International Journal (SIPIJ) Vol.3, No.4, August 2012

\section{ACKNOWLEDGEMENTS}

We want to thank Dr. Meena Pangarkar and Dr. W. K. Raut, Govt. Medical College and Hospital, Nagpur, India for providing the medical image data and interpretation for the analysis. Also they help in understanding the complete slide preparation procedure for histology image aquicision.

\section{REFERENCES}

[1] Cigdem Demir And B’Ulent Yener, “Automated Cancer Diagnosis Based On Histopathological Images: A Systematic Survey”, Technical Report, Rensselaer Polytechnic Institute, Department Of Computer Science, Tr-05-09. 1

[2] S. Waheed, R. A. Moffitt, Q. Chaudryl, A. N. Young, and M.D. Wang "Computer Aided Histopathological Classification of Cancer Subtypes", 1-4244-1509-8/07,2007 IEEE.

[3] Olcay Sertel, Umit V. Catalyurek, Hiroyuki Shimada, and Metin N. Gurcan, “ Computer-aided Prognosis of Neuroblastoma: Detection of Mitosis and Karyorrhexis Cells in Digitized Histological Images",31st Annual International Conference of the IEEE EMBS Minneapolis, Minnesota, USA, September 2-6, 2009

[4] Ajay Basavanhallya, Elaine Yu, Jun Xu, Shridar Ganesan, Michael Feldman, John Tomaszewski, Anant Madabhushi, "Incorporating Domain Knowledge for Tubule Detection in Breast Histopathology Using O'Callaghan Neighborhoods”, Medical Imaging 2011: Computer-Aided Diagnosis, Proc. of SPIE Vol. 7963, 796310, doi: 10.1117/12.878092

[5] Jun Xu, Andrew Janowczyk, Sharat Chandran, Anant Madabhushi, "A Weighted Mean Shift, Normalized Cuts Initialized Color Gradient Based Geodesic Active Contour Model: Applications to Histopathology Image Segmentation", Medical Imaging 2010: Image Processing, Proc. of SPIE Vol. 7623, 76230Y, doi: 10.1117/12.845602

[6] H. Fatakdawala, J. Xu, A. Basavanhally, G. Bhanot, S. Ganesan, M. Feldman, J. E. Tomaszewski, and A. Madabhushi, "Expectation maximization driven geodesic active contour with overlap resolution (emagacor): application to lymphocyte segmentation on breast cancer histopathology," Biomedical Engineering, IEEE Transactions on , In Press. 2009 Ninth IEEE International Conference on Bioinformatics and Bioengineering

[7] Jean-Romain Dalle, Wee Kheng Leow, Daniel Racoceanu, Adina Eunice Tutac, Thomas C. Putti, "Automatic Breast Cancer Grading of Histopathological Images", 30th Annual International IEEE EMBS Conference Vancouver, British Columbia, Canada, August 20-24, 2008

[8] Baochuan Pang, Yi Zhang, Qianqing Chen, Zhifan Gao, Qinmu Peng, Xinge You,"Cell Nucleus Segmentation in Color Histopathological Imagery Using Convolutional Networks", 978-1-42447210-9/10, 2010 IEEE

[9] S. Naik, S. Doyle, S. Agner, A. Madabhushi, M. Feldman, and J. Tomaszewski, "Automated gland and nuclei segmentation for grading of prostate and breast cancer histopathology," in Biomedical Imaging: From Nano to Macro, 2008. ISBI 2008. 5th IEEE International Symposium on, pp. 284287, May 2008.

[10] Scott Doyle, Shannon Agner, Anant Madabhushi, Michael Feldman, John Tomaszewski, "Automated Grading Of Breast Cancer Histopathology Using Spectral Clustering with Textural and Architectural Image Features”, 978-1-4244-2003-2/08, 2008 IEEE.

[11] Metin N. Gurcan, Tony Pan, Hiro Shimada, and Joel Saltz, "Image Analysis for Neuroblastoma Classification: Segmentation of Cell Nuclei", Proceedings of the 28th IEEE EMBS Annual International Conference New York City, USA, Aug 30-Sept 3, 2006

[12] Xiaobo Zhou, Fuhai Li, Jun Yan, and Stephen T. C. Wong "A Novel Cell Segmentation Method and Cell Phase Identification Using Markov Model”, IEEE Transactions On Information Technology In Biomedicine, Vol. 13, No. 2, March 2009 
Signal \& Image Processing : An International Journal (SIPIJ) Vol.3, No.4, August 2012

[13] Cigdem Gunduz-Demir, Melih Kandemir, Akif Burak Tosun, Cenk Sokmensuer, "Automatic segmentation of colon glands using object-graphs", Medical Image Analysis 14(2010) 1-12

[14] Yousef Al-Kofahi, Wiem Lassoued, William Lee, and Badrinath Roysam "Improved Automatic Detection and Segmentation of Cell Nuclei in histopathology Images," IEEE Transactions On Biomedical Engineering, Vol. 57, No. 4, April 2010 Pp 841-850

[15] Maciej Hrebień, Jozef Korbicz, and Andrzej Obuchowicz" Hough Transform, Search Strategy and Watershed Algorithm in segmentation of Cytological Images":Computer Recognition Systems 2, ASC 45, pp. 550-557,Springer-Verlag Berlin Heidelberg, 2007

[16] Omar S. Al-Kadi, "Texture measures combination for improved meningioma classification of histopathological images”, Pattern Recognition 43(2010) 2043-2053

[17] T.S. Subashini, V. Ramalingam, S. Palanivel, "Breast mass classification based on cytological patterns using RBFNN and SVM", Expert Systems with Applications, 2008

[18] Olcay Sertel • Jun Kong • Umit V. Catalyurek • Gerard Lozanski • Joel H. Saltz • Metin N. Gurcan , J Sign Process Syst, "Histopathological Image Analysis Using Model-Based Intermediate Representations and Color Texture: Follicular Lymphoma Grading”, DOI 10.1007/s11265-0080201

[19] P. S. Umesh Adiga, B. B. Chaudhari, "Region based techniques for segmentation of volumetric histopathological images", Computer Methods \& Programs in Biomedicine 61(2000) 23-47

[20] Ajay Nagesh Basavanhally, Shridar Ganesan, Shannon Agner, James Peter Monaco, Michael D. Feldman, John E. Tomaszewski, Gyan Bhanot, and Anant Madabhushi, "Computerized ImageBased Detection and Grading of Lymphocytic Infiltration in HER2+ Breast Cancer Histopathology”, IEEE Transactions On Biomedical Engineering, Vol. 57, No. 3, March 2010,Pp $642-53$

[21] Pornchai Phukpattaranont and Pleumjit Boonyaphiphat, Non-members "Color Based Segmentation of Nuclear Stained Breast Cancer Cell Images”, ECTI Transactions On Electrical Eng., Electronics and Communications Vol.5, No.2 August 2007

[22] M. Muthu Rama Krishnan, Mousumi Pal, Suneel K Bomminayuni, Chandan Chakraborty, Ranjan Rashmi Paul, Jyotirmoy Chatterjee, Ajoy K Roy, "Automated classification of cells in sub-epithelial connective tissue of oral sub-mucous fibrosis - An SVM based approach", Computers in Biology \& Medicine 39(2009) 1096-1104

[23] Olcay Sertel, Umit V. Catalyurek, Gerard Lozanski, Arwa Shanaah, Metin N. Gurcan, “ An Image Analysis Approach for Detecting Malignant Cells in Digitized H\&E-stained Histology Images of Follicular Lymphoma”, 1051-4651/10, 2010 IEEE, DOI 10.1109/ICPR.2010.76

[24] M Muthu Rama Krishnan, Pratik Shaht, Madhumala Ghosh, Mousumi Pal, Chandan Chakraborty, Ranjan R Paul, Jyotirmoy Chatterjee and Ajoy K. Ray, "Automated Characterization of Subepithelial Connective Tissue Cells of Normal Oral Mucosa: Bayesian Approach", Proceedings of the 2010 IEEE Students' Technology Symposium 3-4 April 2010, IIT Kharagpur

[25] Scott Doyle, Michael Feldman, John Tomaszewski, and Anant Madabhushi, "A Boosted Bayesian Multi-Resolution Classifier for Prostate Cancer Detection from Digitized Needle Biopsies", Transactions On Biomedical Engineering

[26] M. Murat Dundar, Sunil Badve, Gokhan Bilgin, Vikas Raykar, Rohit Jain, Olcay Sertel, and Metin N. Gurcan, "Computerized Classification of Intraductal Breast Lesions Using Histopathological Images”, IEEE Transactions On Biomedical Engineering, Vol. 58, No. 7, July 2011, pp. 1977-84.

[27] Akif Burak Tosun, Cigdem Gunduz-demir, "Graph Run-length Matrices For Histopathological Image Segmentation”, IEEE Transactions On Medical Imaging, Vol. 30, No. 3, March 2011, pp. 721-31.

[28] Hui Kong, Metin Gurcan, and Kamel Belkacem-Boussaid, "Partitioning Histopathological Images: An Integrated Framework for Supervised Color-Texture Segmentation and Cell Splitting", IEEE Transactions On Medical Imaging, Vol. 30, No. 9, September 2011, pp. 1661-77 
Signal \& Image Processing : An International Journal (SIPIJ) Vol.3, No.4, August 2012

[29] Frank Y. Shih, Shouxian Cheng, “Automatic seeded region growing for color image segmentation”, Image and Vision Computing 23 (2005) 877-886, doi:10.1016/j.imavis.2005.05.015

[30] Tie Qi Chen, Yi Lu, “Color image segmentation-an innovative approach”, Pattern Recognition 35 (2002) 395-405

[31] Dogan Aitunbay, Celal Cigir, Cenk Sokmensuer and Cigdem Gunduz-Demir, "Color Graphs for Automated Cancer Diagnosis and grading", IEEE Transaction on Biomedical Engineering, Vol. 57, No. 3, March 2010.

[32] Akif Burak Tosun, Melih Kandemir, Cenk Sokmensure, Cigdem Gunduz-demir, "Object-oriented texture analysis for the unsupervised segmentation of biopsy images for cancer detection", Pattern Recognition 42 (2009) 1104-1112,doi:10.1016/j.patcog.2008.07.007.

[33] R.C. Gonzalez, R.F. Woods, "Digital Image Processing”, 3rd ed. Pearson Prentice Hall, 2008.

[34] M. Gurcan, L. Boucheron, A.Can,A. Madabhushi, N. Rajpoot, B. Yener, "Histopathological image analysis: a review", IEEE Reviews in Biomedical Engineering2(2009).

[35] C. G. Loukas, A. Linney, "A survey on histological image analysis-based assessment of three major biological factors influencing radiotherapy: proliferation, hypoxia and vasculature", Computer Methods and Programs in Biomedicine 74(3) (2004) 183-199. 